\title{
Simplicial Decomposability
}

\author{
DAVID COOK II
}

\begin{abstract}
We introduce a new Macaulay2 package, SimplicialDecomposability, which works in conjunction with the extant package SimplicialComplexes in order to compute a shelling order, if one exists, of a specified simplicial complex. Further, methods for determining vertex-decomposability are implemented, along with methods for determining $k$-decomposability.
\end{abstract}

IntRoduction. Given a finite vertex set $V$, a simplicial complex $\Delta$ is a set of subsets of $V$ such that $\tau \in \Delta$ whenever $\tau \subset \sigma$ for some $\sigma \in \Delta$ and such that $\{v\} \in \Delta$ for all $v \in V$. The elements $\sigma \in \Delta$ are called faces or simplices and the maximal faces, i.e., those not contained in any other face, are called facets. The dimension of the face $\sigma$ is $\# \sigma-1$ and the dimension of $\Delta$ is $\max \operatorname{dim} \sigma$. Let $d=\operatorname{dim} \Delta+1$. The $f$-vector of $\Delta$ is the $(d+1)$-tuple $\left(f_{-1}, \ldots, f_{d-1}\right)$, where $f_{i}$ is the number of faces of dimension $i$ in $\Delta$. Using this, the $h$-vector of $\Delta$ is the $(d+1)$-tuple $\left(h_{0}, \ldots, h_{d}\right)$ given by $h_{j}=\sum_{i=0}^{j}(-1)^{j-i}\left(\begin{array}{c}d-i \\ j-i\end{array}\right) f_{i-1}$ for $0 \leq j \leq d$.

Given a field $K$, let $K[V]$ be the polynomial ring with variables indexed by the vertices $V$. The Stanley-Reisner ideal of $\Delta$ is the ideal $I(\Delta)$ in $K[V]$ generated by the minimal non-faces of $\Delta$ and the Stanley-Reisner ring of $\Delta$ is the ring $K[\Delta]=K[V] / I(\Delta)$. Thus the Stanley-Reisner ideals of complexes on a given vertex set $V$ are exactly the squarefree monomial ideals in $K[V]$. Given the relations between the complex and the ideal, one can use tools from both algebra and combinatorics to study properties of both. For example, the $h$-vector of a complex $\Delta$ is the coefficient-vector of the numerator of the Hilbert series of $K[\Delta]$.

The package SimplicialComplexes by Sorin Popescu, Gregory G. Smith, and Mike Stillman already implements many methods for simplicial complexes in Macaulay2 [M2], a software system designed to aid in research of commutative algebra and algebraic geometry. We introduce a new package, SimplicialDecomposability, for Macaulay 2 which provides several new methods for testing various forms of decomposability for simplicial complexes. Particularly, the package implements methods for testing shellability and vertex-decomposability.

ShELLABILITy. Given a finite set $\sigma$, let $2^{\sigma}$ be the set of all subsets of $\sigma$. Let $\Delta$ be a simplicial complex that has equidimensional facets, i.e., is pure. Then by Definition III.2.1 in [S], $\Delta$ is shellable if its facets can be ordered $\sigma_{1}, \ldots, \sigma_{n}$ so that $\bigcup_{j=1}^{i} 2^{\sigma_{j}} \backslash \bigcup_{j=1}^{i-1} 2^{\sigma_{j}}$ has a unique minimal element for $2 \leq i \leq n$, such an ordering is called a shelling order. See Definition 2.1 in [BW1] for the definition of non-pure shellability, which is implemented in the package for non-pure complexes.

Shellability is of interest because it implies a number of nice properties. In particular, if a pure simplicial complex is shellable, then its Stanley-Reisner ring is Cohen-Macaulay over every field [S, Theorem III.2.5]. Moreover, its $h$-vector is non-negative and can be read off from any shelling

2010 Mathematics Subject Classification. 05E45, 13 F55.

SimplicialDecomposability version 1.0.5. 
order [S, Theorem III.2.3]. Further still, the $h$-vectors of shellable pure complexes are numerically characterized [S, Theorems II.2.2 and II.3.3].

We recall that the Alexander dual of a simplicial complex $\Delta$ on vertex set $V$ is the simplicial complex $\Delta^{\vee}:=\{V \backslash F \mid F \notin \Delta\}$. Further, we say an ideal $I$ has linear quotients if the minimal generators of $I$ can be ordered $f_{1}, \ldots, f_{n}$ such that for $2 \leq i \leq n$, the quotient ideal $\left(f_{1}, \ldots, f_{i-1}\right):\left(f_{i}\right)$ is generated by linear forms, in this case the sequence $\left\{\left(f_{1}\right):\left(f_{2}\right),\left(f_{1}, f_{2}\right):\left(f_{3}\right), \ldots,\left(f_{1}, \ldots, f_{n-1}\right): f_{n}\right\}$ is called a linear quotient order of $I$ with respect to $f_{1}, \ldots, f_{n}$.

In the following example we demonstrate Theorem 1.4(c) of [HHZ], which shows that a pure simplicial complex is shellable if and only if the Stanley-Reisner ideal of the Alexander dual has linear quotients. We begin by constructing the polynomial ring $R=\mathbb{Q}[a, b, c, d, e, f, g]$ and a simplicial complex $D$, which we verify is pure. Loading the package SimplicialDecomposability automatically loads the package Simplicialcomplexes.

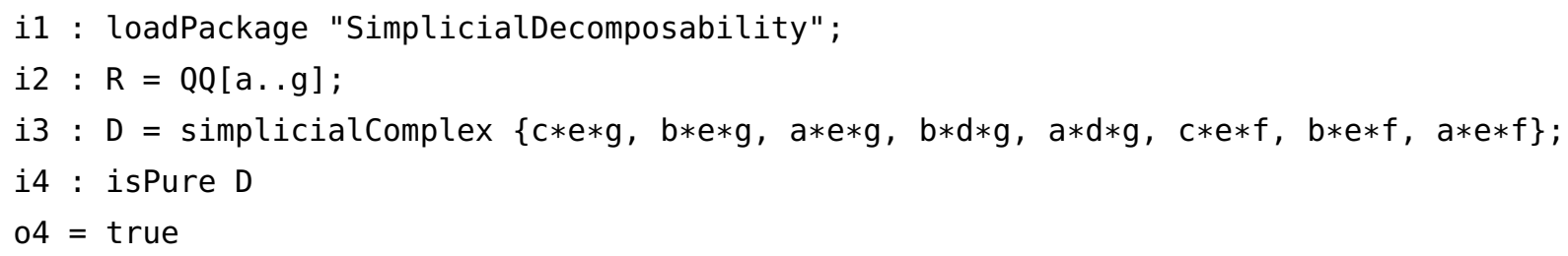

We can recover a sequence of linear quotients directly from a shelling order. We recall that a pure simplicial complex $\Delta$ is shellable if there is an order of the facets $F_{1}, \ldots, F_{n}$ such that for $0<j<i$ there exists an $x \in F_{i} \backslash F_{j}$ and a $0<k<i$ such that $F_{i} \backslash F_{k}=\{x\}$. The set of vertices associated to each $i$ in the preceding statement generate the linear quotient order of $I\left(\Delta^{\vee}\right)$ with respect to the given shelling order (see the proof of Theorem 1.4(c) in [HHZ]).

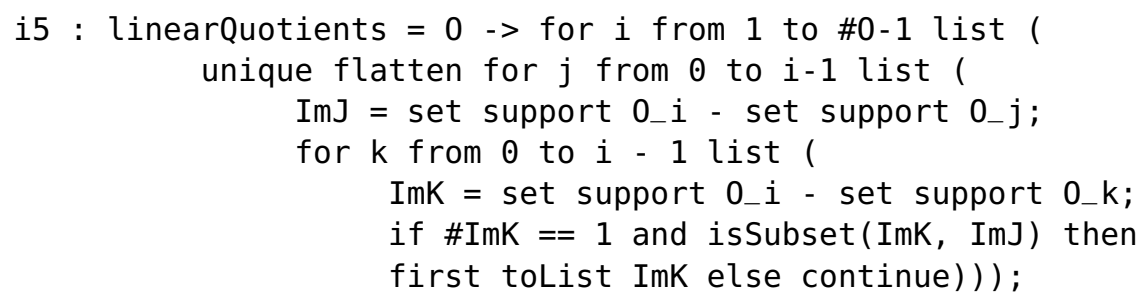

We generate a shelling order $O_{1}$ of $D$ with the method shelling0rder. This method attempts to build up a shelling order of $D$ recursively using a depth-first search, adding one facet at a time. We note that in the non-pure case, the method only searches the remaining facets of largest dimension.

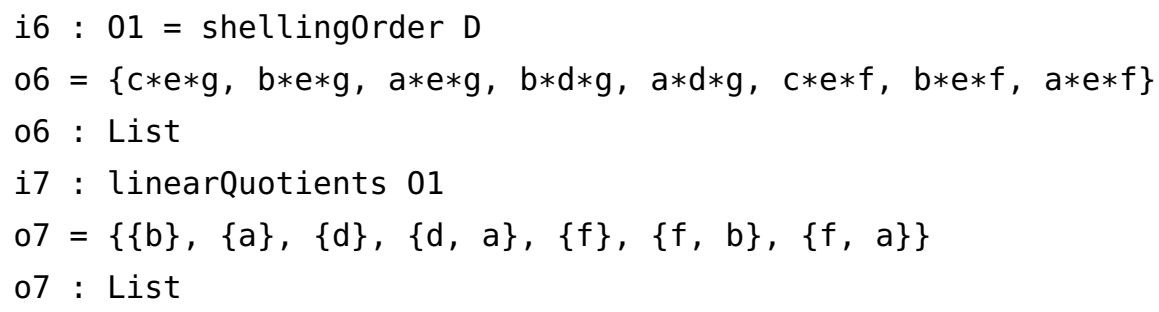

It is sometimes beneficial to have more than one shelling order for a given simplicial complex. We can use the option Random with the method shelling0rder to first apply a random permutation to the facets before preceding with the recursion.

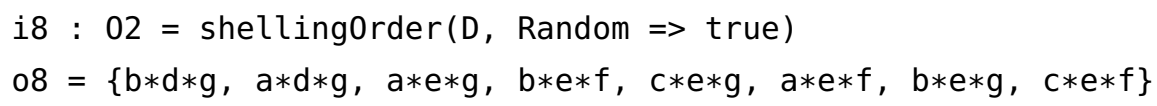




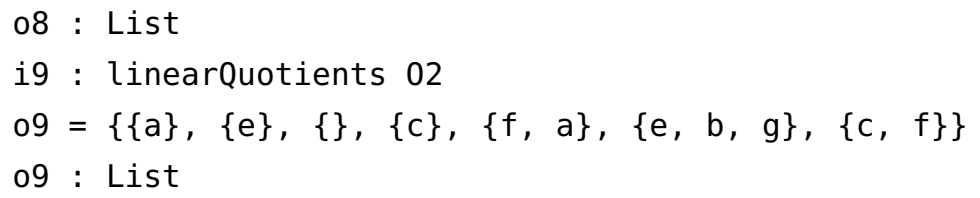

Alternately, we may use the option Permutation with the method shelling0rder to force a given permutation on the facets before preceding with the recursion.

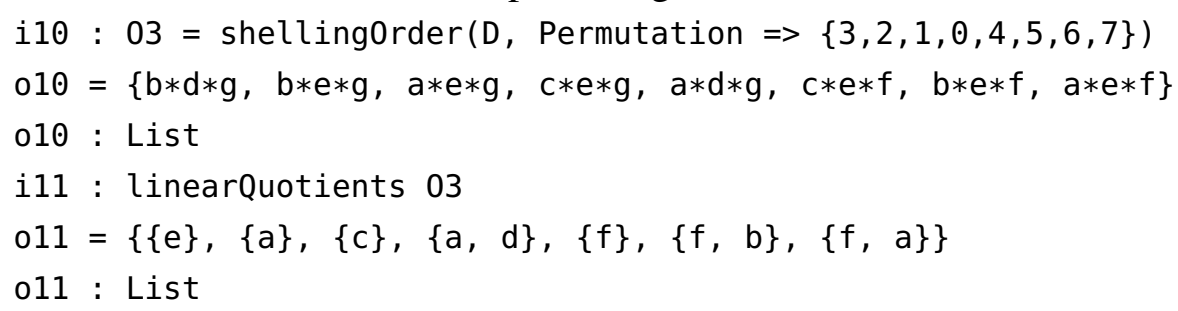

Thus we now have multiple linear quotient orders associated to the ideal $I\left(D^{\vee}\right)$, each coming from a shelling order of $D$.

VERTEX-DECOMPOSABILITY. Let $\Delta$ be a pure simplicial complex and $\sigma$ a face of $\Delta$. Then the link and face deletion of $\sigma$ in $\Delta$ are the simplicial complexes

$$
\operatorname{link}_{\Delta} \sigma:=\{\tau \in \Delta \mid \sigma \cap \tau=\emptyset, \sigma \cup \tau \in \Delta\} \text { and } \operatorname{del}_{\Delta} \sigma:=\{\tau \in \Delta \mid \sigma \nsubseteq \tau\} .
$$

Definition 2.1 in [PB] defines $\Delta$ to be vertex-decomposable if either $\Delta$ is a simplex or there exists a vertex $x \in \Delta$, called a shedding vertex, such that $\operatorname{link}_{\Delta} x$ and $\operatorname{del}_{\Delta} x$ are vertex-decomposable.

See Definition 11.1 in [BW2] for the definition of non-pure vertex-decomposability, which is implemented in the package for non-pure complexes. Also, see Definitions 3.1 and 3.6 in [W] for the generalization of vertex-decomposability, called $k$-decomposability. It is implemented in the package with the methods iskDecomposable and issheddingFace.

Being vertex-decomposable is a strong property which implies many things. A pure vertexdecomposable simplicial complex is shellable [PB, Theorem 2.8] and hence has non-negative $h$-vector [S, Theorem III.2.3] and its Stanley-Reisner ring is Cohen-Macaulay [S, Theorem III.2.5]. Furthermore, the $h$-vectors are numerically characterised for vertex-decomposable simplicial complexes [L, Theorem 3.5]. Moreover, the Stanley-Reisner ring of a pure vertex-decomposable complex is squarefree glicci [NR, Definition 2.2 and Theorem 3.3].

In the following example we demonstrate that the simplicial complex $D$ from the previous example is indeed squarefree glicci. We use [NR, Remark 2.4] to find a basic double link of $I(D)$ to $I\left(\operatorname{link}_{D} v\right)$, both in $R$, for some shedding vertex $v$ of $D$.

First, we verify that $D$ is vertex-decomposable. Then we find its shedding vertices.

i12 : isvertexDecomposable $D$

012 = true

i13 : $\operatorname{select}(\operatorname{allFaces}(\theta, D), v \rightarrow$ isSheddingVertex( $v, D)$ )

$013=\{a, b, c, d, f\}$

013 : List

We choose the shedding vertex $f$ of $D$ and generate $E=\operatorname{link}_{D} f$. Then we find its shedding vertices.

i14: $E=\operatorname{link}(D, f)$;

i15 : ideal $\mathrm{E}$ 


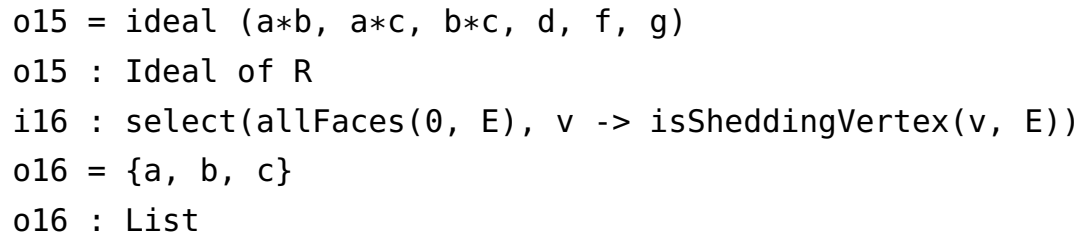

We now choose the shedding vertex $c$ of $E$ and generate $F=\operatorname{link}_{E} c$. Notice then that the StanleyReisner ideal of $F$ is a complete intersection.

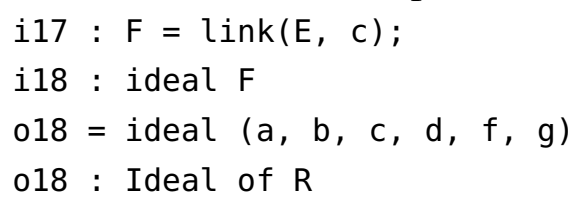

Hence, we now have the following sequence of basic double links in $R$ which has squarefree terms on the even steps (the odd steps are omitted):

$$
\mathbb{Q}[D]=(a b, a c, b c, c d, d e, d f, f g) \sim \mathbb{Q}[E]=(a b, a c, b c, d, f, g) \sim \mathbb{Q}[F]=(a, b, c, d, f, g) .
$$

ACKNOWLEDGEMENTS. The author would like to thank his advisor, Uwe Nagel, for reading drafts of this article and making comments thereon. The author would also like to thank Russ Woodroofe for pointing out the non-pure generalisation of $k$-decomposability in [W] and an anonymous referee for inspiring a more concrete example herein.

Part of the work for this paper was done while the author was at a Macaulay 2 workshop in Berkeley, California, January 8, 2010 through January 12, 2010, organised by Amelia Taylor and Hirotachi Abo with David Eisenbud, Daniel R. Grayson, and Michael E. Stillman, and funded by the National Security Agency (NSA) through grant H98230-09-1-0111.

\section{REFERENCES.}

[BW1] A. Björner and M.L. Wachs, Shellable nonpure complexes and posets. I, Trans. Amer. Math. Soc. 348 (1996), no. 4, $1299-1327$.

[BW2] __ Shellable nonpure complexes and posets. II, Trans. Amer. Math. Soc. 349 (1997), no. 10, 3945 - 3975.

[HHZ] J. Herzog, T. Hibi, and X. Zheng, Dirac's theorem on chordal graphs and Alexander duality, European J. Combin. 25 (2004), no. 7, 949 - 960.

[L] C.W. Lee, Two combinatorial properties of a class of simplicial polytopes, Israel J. Math. 47 (1984), no. 4, $261-269$.

[M2] D.R. Grayson and M.E. Stillman, Macaulay 2, a software system for research in algebraic geometry, available at www. math. uiuc. edu/Macaulay2/.

[NR] U. Nagel and T. Römer, Glicci simplicial complexes, J. Pure Appl. Algebra 212 (2008), no. 10, 2250 - 2258.

[PB] J.S. Provan and L.J. Billera, Decompositions of simplicial complexes related to diameters of convex polyhedra, Math. Oper. Res. 5 (1980), no. 4, 576 - 594.

[S] R.P. Stanley, Combinatorics and commutative algebra, 2nd ed., Progress in Mathematics, vol. 41, Birkhäuser Boston Inc., Boston, MA, 1996.

[W] R. Woodroofe, Chordal and sequentially Cohen-Macaulay clutters, available at arXiv: 0911.4697v2 [math. c0].

$$
\text { ReCEIVED : 2010-02-18 REvised : 2010-06-10 ACCEPTED : 2010-08-03 }
$$

